\title{
Teil-Berufsausübungsgemeinschaften
}

\section{Ist die Berufsordnung verfassungswidrig?}

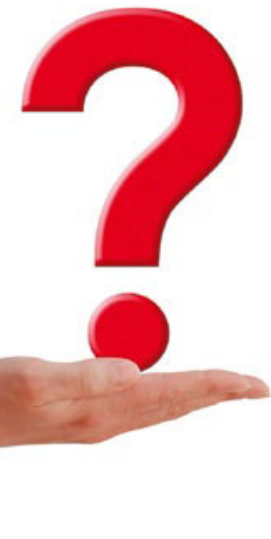

Die Berufsordnung für Ärzte könnte teilweise verfassungswidrig sein. So zumindest lautet das Urteil eines Landgerichts. Im konkreten Fall sahen die Richter einen Radiologen benachteiligt, der mit anderen niedergelassenen Ärzten kooperieren wollte. Dabei ist der Fall heikler als gedacht: Denn der fragliche Passus steckt auch in der neuen Musterberufsordnung.

F ür seine Brisanz ist nachfolgender Fall (Aktenzeichen 3 O 13/10) bislang in der Öffentlichkeit eher stiefmütterlich behandelt worden: Das Landgericht Mosbach hat in einem Verfahren eine Passage in der Musterberufsordnung (MBO) für Ärzte entdeckt, die gegen das Grundgesetz verstößt. In dem Verfahren wurde versucht, eine ärztliche Kooperation aufzulösen. Das Gericht bemängelt den Passus der MBO, der Teil-Berufsausübungsgemeinschaften (TBAG) oder Teilgemeinschaftspraxsen mit Ärzten, die innerhalb der Kooperation lediglich medizinisch-technische Leistungen auf Veranlassung der übrigen Partner erbringen, mehr oder weniger verbietet. Das schränke die Berufsfreiheit der Ärzte ein, so die Richter.

Den Stein ins Rollen gebracht hat die „Zentrale zur Bekämpfung unlauteren Wettbewerbs e.V.“. Diese klagte gegen eine privatärztliche TBAG zwischen Allgemeinärzten und Radiologen in BadenWürttemberg. Der Vorwurf: Die Radiologen würden innerhalb der TBAG rein medizinisch-technische Leistungen und diese nur auf Veranlassung ihrer hier allgemeinärztlichen Partner erbringen. Das verstoße gegen die Berufsordnung für Ärzte und sei eine Umgehung des $\$ 31$, der unerlaubte Zuweisungen gegen Entgelt verbietet. Daher sollten die Ärzte die TBAG auflösen.
Das sahen die Richter am LG Mosbach anders. Der Verstoß liege nicht bei den Ärzten, sondern in der Berufsordnung. Denn $₫ 18$ Abs. 1 Satz 2 Alternative 1 der Berufsordnung der Landesärztekammer Baden-Württemberg sei nicht mit dem Grundgesetz vereinbar. In dem Passus steht nämlich wörtlich: „Der Zusammenschluss zur gemeinsamen Ausübung des Arztberufs kann zum Erbringen einzelner Leistungen erfolgen, sofern er nicht lediglich einer Umgehung des $₫ 31$ dient. Eine Umgehung liegt insbesondere vor, wenn sich der Beitrag der Ärztin oder des Arztes auf das Erbringen medizinisch-technischer Leistungen auf Veranlassung der übrigen Mitglieder einer Teil-Berufsausübungsgemeinschaft beschränkt ..."Nach Ansicht der Richter würden Radiologen hier benachteiligt, deren klassisches Betätigungsfeld nun einmal gerade solche Leistungen seien und dies in der Regel auf Veranlassung anderer Ärzte. Radiologen seien, egal ob sie ihren Beruf in einer BAG ausüben oder nicht, davon abhängig, dass ihnen Patienten von anderen Ärzten zugewiesen werden, heißt es im Urteil. Konkret bedeute dies, so die Richter, dass Radiologen keine BAG mit zuweisenden Fachgruppen eingehen dürften. Und das sei nicht mit $₫ 12$ des Grundgesetzes vereinbar, der auch das Recht, sich beruflich zusammenzuschließen, schütze. Zumal

\section{Versto $ß$ in der Berufsordnung}

die reine Zuweisung von Patienten an die Radiologen noch nicht dazu berechtige, ihnen oder den Allgemeinärzten zu unterstellen, es würde hierfür ein Entgelt fließen.

Selbst die Tatsache, dass der Gesellschaftsvertrag der TBAG vorsieht, dass ein Prozent des Gewinns vorab nach Köpfen verteilt wird, brachte das Gericht nicht von seiner Meinung ab. Denn es gebe einen Grund für diese Gewinnverteilung: Die Partner wollten damit einen ideellen Anteil an der gemeinsamen Leistung auch gemeinsam verteilen. Die Ärzte führen auch das Argument ins Feld, dass in anderen Kooperationsformen, etwa in MVZ, durchaus auch Radiologen beteiligt sein dürfen - die TBAG werde daher durch die Regelung benachteiligt.

\section{Auch andere Kammergebiete sind betroffen}

Interessant ist dieses Urteil auch für andere Kammergebiete insofern, als dass jener Passus in $\$ 18$ der Berufsordnung in Baden-Württemberg genau so in der MBO für Ärzte steht. Das heißt, diese Landesärztekammer hat sich hier an den Vorgaben der Bundesärztekammer orientiert, und das dürften auch die anderen so gemacht haben. Auch die neue Version der MBO, in der insgesamt 20 Paragrafen der MBO umgeschrieben oder erweitert wurden, wird vorerst noch diesen Passus zu den TBAG enthalten.

Nachbessern will die Landesärztekammer Baden-Württemberg aber erst einmal nicht, erklärt Pressesprecher Dr. Oliver Erens auf Nachfrage. Der Grund: Das Urteil des LG Mosbach ist noch nicht rechtskräftig, weil die Wettbewerbszentrale beim OLG Karlsruhe bereits Berufung eingelegt hat. Die Kammer will hier die Entscheidung des OLG abwarten. Und auch ob die MBO nachgebessert wird, ist noch offen.

Rebekka Höhl 